\title{
Salud mental en funcionarios de una universidad chilena: desafíos en el contexto de la COVID-19
}

\author{
Ricardo Jorquera Gutiérrez \\ Universidad de Atacama, Copiapó, Chile \\ https://orcid.org/0000-0002-7059-8488 \\ Fernando Herrera Gallardo \\ Universidad de Atacama, Copiapó, Chile \\ https://orcid.org/0000-0002-4941-1930
}

Recibido: 01/10/20 Revisado: 04/11/20 Aceptado: 11/11/20 Publicado: 05/12/20

\begin{abstract}
Resumen
El presente estudio tuvo como objetivo describir los niveles de estrés, depresión y ansiedad en funcionarios de una universidad del norte de Chile, en el contexto de la pandemia de la COVID19. Asimismo, buscó establecer la relación de esta sintomatología con agotamiento y sobrecarga laboral, además de diversas variables sociodemográficas y laborales. Los resultados muestran la presencia de estrés en un 55,7\% de los funcionarios, depresión en un $26 \%$ de ellos y ansiedad en un $29,2 \%$. Los mayores índices de estrés se observan en mujeres, académicos, menores de 40 años, y en trabajadores contratados. Se verificó empíricamente el ajuste de un modelo predictivo que permitió evidenciar que la sobrecarga laboral percibida explica el agotamiento laboral, y, a su vez, esta variable predice el estrés evidenciado por los funcionarios de la Universidad. Se concluye analizando las implicancias de los resultados en la gestión de instituciones de educación superior, considerando el actual contexto pandémico.
\end{abstract}

Palabras clave: Académicos; estrés; agotamiento laboral; sobrecarga laboral; COVID-19.

\section{Mental health in officials of a chilean university: challenges in the context of COVID-19}

\begin{abstract}
The present study aimed to describe the levels of stress, depression and anxiety in workers at a university in northern Chile, in the context of COVID-19 pandemic. Likewise, it sought to establish the relationship of this symptomatology with exhaustion and work overload, in addition to various sociodemographic and work variables. The results show the presence of stress in $55.7 \%$ of the employees, depression in $26 \%$ of them and anxiety in $29.2 \%$. The highest rates of stress are observed in women, academics, those under 40 years of age, and in contract workers. The adjustment of a predictive model was empirically verified to show that the perceived work overload allows to explain the work burnout, and, in turn, this variable predicts the stress evidenced by the University workers. It concludes by analyzing the implications of the results in the management of higher education institutions, considering the current pandemic context.
\end{abstract}

Keywords: Academics; stress; job burnout; work overload; COVID-19 


\title{
Saúde mental em funcionários de uma universidade chilena: desafios no contexto de COVID-19
}

\begin{abstract}
Resumo
O presente estudo teve como objetivo descrever os níveis de estresse, depressão e ansiedade em trabalhadores de uma universidade no norte do Chile, no contexto de COVID-19. Da mesma forma, buscou estabelecer a relação dessa sintomatologia com o esgotamento e a sobrecarga de trabalho, além de diversas variáveis sociodemográficas e laborais. Os resultados mostram a presença de estresse em $55,7 \%$ dos funcionários, depressão em $26 \%$ deles e ansiedade em $29,2 \%$. As maiores taxas de estresse são observadas em mulheres, acadêmicas, menores de 40 anos e em trabalhadores contratados. 0 ajuste de um modelo preditivo foi verificado empiricamente para mostrar que a sobrecarga de trabalho percebida permite explicar o burnout e, por sua vez, essa variável prediz o estresse evidenciado pelos trabalhadores da Universidade. Conclui analisando as implicações dos resultados na gestão das instituições de ensino superior, considerando o atual contexto pandêmico.
\end{abstract}

Palavras-chave: Acadêmicos; estresse; Esgotamento do trabalho; sobrecarga de trabalho; COVID-19.

Citar como:

Jorquera , R. \& Herrera, F. (2020). Salud mental en funcionarios de una universidad chilena: desafíos en el contexto de la COVID-19. Revista Digital de Investigación en Docencia Universitaria. 14(2), e1310. https://doi.org/10.19083/ ridu.2020.1310

\section{Introducción}

Las universidades chilenas suspendieron sus actividades presenciales a mediados de marzo de 2020, con fines preventivos, dada la pandemia de la COVID-19. Esta realidad significó un desafío para las comunidades educativas, las cuales ajustaron sus procesos, tanto en los aspectos concernientes a la enseñanza-aprendizaje, como en los de tipo administrativo. Buena parte de estos comenzaron a realizarse a través de sistemas remotos de teletrabajo, lo cual demandó un importante esfuerzo adaptativo. Estos ajustes, propios de la transformación de los sistemas de trabajo, fijan la atención respecto de la posibilidad de que estos cambios produzcan algún efecto adverso sobre la salud psicológica de los funcionarios universitarios.

Los antecedentes iniciales ponen en relieve el efecto que ha tenido la pandemia, y muy especialmente el confinamiento, sobre la salud mental de la población en general. En China, durante los primeros meses de la pandemia se encontró que un $53,8 \%$ de las personas que participaron de un estudio manifestaba algún impacto psicológico producto de la COVID-19, entre los que destaca la sintomatología depresiva, ansiosa y estrés (Wang et al., 2020). Igual panorama se observó en el norte de España, donde se constató un incremento en este tipo de sintomatología producto del confinamiento (Ozamiz-Etxebarria et al., 2020). En el caso de Latinoamérica, en Argentina se ha constatado un aumento de la ansiedad fóbica, comparando estudios efectuados entre los años 2003 y 2020, siendo las más afectadas las mujeres (Vela et al., 2020).

Uno de los aspectos que cobra relevancia estudiar en el contexto actual, hace referencia al impacto que han tenido las transformaciones del trabajo universitario desde un formato presencial a un trabajo de tipo remoto.

En Chile se ha podido observar que el cambio a modalidad de teletrabajo ha implicado una disminución en el engagement (energía, vigor y dedicación) y un aumento en los niveles de agotamiento (sensación de desgaste y fatiga crónica asociados al estrés) de los trabajadores. Este efecto ha sido facilitado por un incremento en las horas de trabajo, condiciones laborales inadecuadas (falta de espacios adecuados y herramientas tecnológicas para llevarlo a cabo), la necesidad de conciliar el trabajo y asuntos propios del hogar y la familia, y la exposición a las noticias (Circular HR, 2020). 
Respecto de lo anterior, la Organización Internacional del Trabajo (2019), antes de la pandemia había mostrado efectos ambiguos e incluso contradictorios del teletrabajo. Si bien había observado aspectos positivos como menores tiempos de desplazamiento, mayor autonomía en la organización del tiempo de trabajo, mejor conciliación general entre la vida laboral y personal y mayor productividad, también se había observado desventajas como la prolongación de las horas de trabajo, una superposición entre el trabajo y la vida personal y una intensificación del trabajo. Sumado a ello, daba cuenta de riesgos para la salud y el bienestar, asociados con asuntos ergonómicos y con factores de riesgo psicosociales, entre ellos, el estrés. Esto iba en la línea de otros estudios que han observado que el teletrabajo puede producir un impacto psicológico en términos emocionales en teletrabajadores, quienes podrían experimentar una mayor sintomatología de estrés, comparado con trabajadores de oficina (Mann y Holdsworth, 2003). A su vez, en el actual panorama de confinamiento, producto de la pandemia, el teletrabajo ha implicado desarrollar las funciones compartiendo espacios con la familia. Es relevante señalar esto, pues la literatura ya había mencionado que compartir el espacio del hogar mientras se trabaja afecta negativamente el tiempo familiar, siendo esta una variable determinante en el conflicto familia-trabajo (Salazar, 2016).

En los últimos años, se ha manifestado una creciente preocupación por la salud mental en el contexto educativo. Asimismo, se ha observado que la indisciplina, la falta de interés de los alumnos y la escasa colaboración de las familias son las principales fuentes de estrés en docentes, siendo esta variable, junto con el grado de satisfacción y compromiso, los mejores predictores de salud mental en profesores (Guerrero-Barona et al., 2018). En otros estudios, profesores chilenos han reconocido como los principales factores que impactan su salud mental: el liderazgo deficiente, los nuevos roles docentes, el agobio laboral, el número de estudiantes en el aula, la desmotivación de los estudiantes y la desvinculación de los padres de su rol educativo (Rubio-González et al., 2019). Por su parte, Jorquera-Gutiérrez et al. (2014) detectaron la relación entre estrés crónico y ausentismo laboral en docentes, evidenciando además una correlación negativa entre los niveles de agotamiento emocional de los equipos de profesores y los resultados obtenidos por sus estudiantes en pruebas estandarizadas. Esto se profundiza al considerar que los profesores tienden a priorizar el desempeño profesional por sobre el cuidado de su salud laboral, percibiendo los factores que regulan la salud laboral como externos y fuera del control personal (Cuadra Martínez et al., 2015). También se ha constatado la relación entre estrés crónico y resiliencia, observándose que los docentes más resilientes logran sobreponerse mejor a las dificultades, mientras que los docentes menos resilientes manifiestan mayor cansancio e indiferencia en el trabajo, en donde algunas variables personales (ej., humor, empatía, personalidad resistente, autoeficacia) permiten hacer frente a las demandas laborales y actúan como factores de protección frente al burnout (García \& Gambarte, 2019).

En el caso particular de la labor académica universitaria, Sánchez y Clavería (2005), en profesores universitarios españoles, evidenciaron relación entre estrés laboral, niveles de satisfacción y salud. Destaca de sus resultados, que el cansancio emocional y la despersonalización fueron más altas en profesores noveles y en los de menor antigüedad en la institución, por lo que concluyeron que a mayor edad disminuirían estos factores. Rodríguez-Martínez, Tovalin-Ahumada, Gil-Monte, Salvador-Cruz y Acle-Tomasini (2018) constataron que el trabajo emocional y los estresores laborales predicen el incremento de síntomas de ansiedad y depresión en un grupo de profesores universitarios. Asimismo, sus resultados mostraron que ser mujer también sería un predictor de la ansiedad, más no de la depresión. Cladellas-Pros, Castelló-Tarrida y Parrado-Romero (2018) evidenciaron que la estabilidad laboral tiene repercusiones en la salud y calidad de vida laboral de profesores universitarios que se encuentran en situación de inestabilidad. En este sentido, profesores de tiempo completo con contratos laborales temporales muestran una peor salud física y mental, mayores síntomas de estrés y una menor satisfacción laboral, en comparación con profesores a tiempo completo y contrato estable o profesores de tiempo parcial. Ilaja y Reyes (2016) encontraron, en profesores universita- 
rios ecuatorianos, que las tareas de investigación y vinculación se relacionan significativamente con burnout y que la actividad de docencia no presenta asociación con este tipo de estrés, lo que podría estar relacionado con una percepción de mayor dominio de esta labor, mientras que la vinculación y la investigación son observadas como una exigencia sin disponer de los recursos para realizarla.

Los anteriores estudios ponen en relevancia el problema de la salud mental de los docentes universitarios desde antes de la pandemia. Dados los cambios que ha tenido las transformaciones del trabajo en instituciones universitarias, a consecuencia de la propagación de la COVID-19, es posible suponer una agudización de este problema. Considerando este particular contexto es que surge el presente estudio, el cual viene a responder a las siguientes preguntas: ¿Cuál es el nivel de estrés, depresión y ansiedad en funcionarios académicos y no académicos de una universidad del norte de Chile? ¿Con qué variables sociodemográficas y laborales se asociaría la presencia de sintomatología psicológica? ¿Cuál es la relación entre la sintomatología psicológica y el agotamiento laboral y la sobrecarga de trabajo percibida?. Consistente con estas preguntas se ha establecido los siguientes objetivos: 1. Describir el nivel de estrés, depresión y ansiedad en funcionarios académicos y no académicos en una universidad pública del norte de Chile. 2. Establecer la relación entre sintomatología psicopatológica con diversas variables sociodemográficas y laborales. 3. Establecer la relación entre los niveles de sintomatología psicopatológica con agotamiento laboral y sobrecarga de trabajo percibidas.

De esta forma, la relevancia de la presente investigación está relacionada con poder establecer la prevalencia de sintomatología psicológica en trabajadores de una institución de educación superior, constatando algunas relaciones con variables sociodemográficas y laborales. Con esto, se espera que la información presente sea un insumo adecuado para la gestión de personas en el contexto de los cambios sociales que ha provocado la COVID-19, favoreciendo la toma de decisiones orientada a atender a las necesidades de académicos, administrativos y otros trabajadores del ámbito universitario.

\section{Método}

Se utilizó una metodología cuantitativa de tipo correlacional, en cuanto se buscó caracterizar las variables del estudio y determinar el grado de relación entre ellas (Hernández \& Mendoza, 2018).

\section{Diseño}

El diseño de investigación utilizado fue no experimental de tipo transversal correlacional. Es preciso señalar que en la investigación no experimental no existe manipulación deliberada de variables, y solo se observan los fenómenos en su ambiente natural para después analizarlos (Hernández, Fernández y Baptista, 2014). Por su parte, la presente investigación es transversal en cuanto se procuró realizar una descripción del nivel de ansiedad, depresión y estrés de los funcionarios de la universidad estudiada y su relación con variables sociodemográficas y laborales, y agotamiento laboral y sobrecarga de trabajo percibidas en un único momento.

\section{Participantes}

Se usó un muestreo no probabilístico de tipo intencional. En el estudio participaron 192 funcionarios académicos y no académicos de una universidad pública del norte de Chile. Un 50,5\% (n=97) eran hombres y un 49,5\% ( $n=95)$ mujeres. Un 63\% eran académicos $(n=121)$ y un 37\% $(n=71)$ funcionarios no académicos (administrativos, personal de apoyo, etc.). Según edad, un 6,8\% $(\mathrm{n}=13)$ tenían entre 20 y 29 años, un 40,1\% ( $n=77)$ entre 30-39 años, un $26,6 \%(n=51)$ entre $40-49$ años, un 17,7\% ( $n=34)$ entre 50-59 años y un 8,9\% (n=17) 60 años o más. Según los años de experiencia laboral en la institución, un $8,9 \%(n=17)$ tenía un año o menos, un 42,2\% ( $\mathrm{n}=81)$ entre 2 y 5 años, un 25\% ( $n=48)$ entre 6 y 10 años, un 13,5\% ( $n=26)$ entre 11 y 20 años y un 10,4\% ( $n=20)$ 21 años o más. Según tipo de contrato, un 61,5\% $(\mathrm{n}=118)$ poseía una condición de contrata (contrato a plazo fijo de carácter anual), un 32,8\% era funcionario a planta (contrato de tipo indefinido), y un $5,7 \%(n=11)$ tenían un contrato a honorarios.

\section{Instrumentos}

\section{Salud Psicológica}

Para estudiar los aspectos referidos a la salud psicológica de los funcionarios académicos y no aca- 
démicos se utilizó el instrumento llamado DASS21, el cual mide depresión, ansiedad y estrés. De forma específica se usó la versión chilena, traducida y adaptada por Vinet, Rehbein, Román y Saiz (2008 en Antúnez \& Vinet, 2012) y modificada por Román (2010 en Antúnez \& Vinet, 2012). Esta escala está conformada por 21 ítems con cuatro alternativas de respuesta tipo Likert. Con respecto a sus características psicométricas Antúnez y Vinet (2012) reportaron una estructura con tres factores que explicaban un 49,99\% de su varianza, y valores de confiabilidad de ,85, 83 y ,73 para la escala de depresión, estrés y ansiedad respectivamente. El presente estudio consideró los puntos de cortes para población general recomendados por Román, Santibáñez y Vinet (2016), quienes establecieron 7(>6) puntos para depresión, 6(>5) para Ansiedad y 7(>6) para estrés.

\section{Agotamiento Laboral}

Esta variable se evaluó mediante la subescala de agotamiento laboral del Cuestionario General de Burnout (Schaufeli et al. 1995, en Moore, 2000). Esta escala posee cinco ítems y un sistema de respuesta tipo Likert de 7 puntos. En el caso de la presente investigación esta escala mostró un alfa de Cronbach de ,94 y una estructura de un factor que explica el 81,7\% de la varianza.

\section{Carga Laboral Percibida}

Esta variable se midió con la Escala de Sobrecarga de Trabajo Percibida de Moore (2000). Esta está formada por 4 reactivos, con un sistema de respuesta tipo Likert de 7 puntos. La escala fue traducida y, en la presente investigación, la escala mostró un alfa de Cronbach de 90 y una estructura unifactorial que explicaba un $77,3 \%$ de la varianza.

\section{Variables sociodemográficas y laborales}

Adicionalmente se incorporó un cuestionario que indagaba acerca de: sexo, edad, tipo de funcionario (académico o no académico), años de experiencia laboral en la institución y tipo de contrato.

\section{Procedimientos}

En primer lugar, se solicitó la autorización de la Dirección Superior de la universidad para realizar la investigación, que acogió positivamente la propuesta. Posteriormente, y considerando la realidad sanitaria, se pidió a los participantes la respuesta de los instrumentos a través de medios electrónicos (formulario de google). A todos ellos se les hizo llegar el enlace a los cuestionarios a sus correos electrónicos institucionales. Previo a la entrega de sus respuestas, leyeron un consentimiento informado y señalaron su aprobación para contestar de forma voluntaria. Las aplicaciones de los instrumentos se efectuaron entre el 11 y el 19 de junio de 2020.

\section{Análisis de datos}

El método de análisis estuvo compuesto por estadística descriptiva a través de cálculo de porcentajes, medidas de tendencia central y de variabilidad. Además, se realizaron asociaciones entre variables categóricas utilizando el estadístico no paramétricos Chi-Cuadrado. Las correlaciones de puntajes se efectuaron a través del coeficiente de correlación de Pearson. Todos estos cálculos se efectuaron a través del software SPSS-22. Adicionalmente, se evaluó empíricamente un modelo predictivo de algunas de las variables de estudio, lo cual se realizó mediante modelamiento de ecuaciones estructurales usando el software AMOS-21.

\section{Resultados}

Los resultados generales muestran presencia de estrés en un 55,7\% de los funcionarios evaluados. Por su parte, fue encontrada sintomatología depresiva en un 26\% y ansiedad en un 29,2\% (ver Figura 1).

Al compararse los niveles de estrés según las variables sociodemográficas y laborales utilizadas, se observa, en primer lugar, diferencias estadísticamente significativas en la presencia de esta variable entre hombres y mujeres (Chi-cuadrado=4,206; $p=, 04)$. En este caso, mientras un 48,5\% de los hombres presentan manifestaciones de estrés, en las mujeres este porcentaje llega a 63,2\%. Según tipo de funcionario, se evidencia un nivel más elevado de estrés en académicos (63,6\%), comparado con funcionarios no académicos (42,3\%) (Chi-cuadrado=8,292; $p=, 004$ ). Según grupos de edad, también se evidencian diferencias estadísticamente significativas en los niveles de estrés 


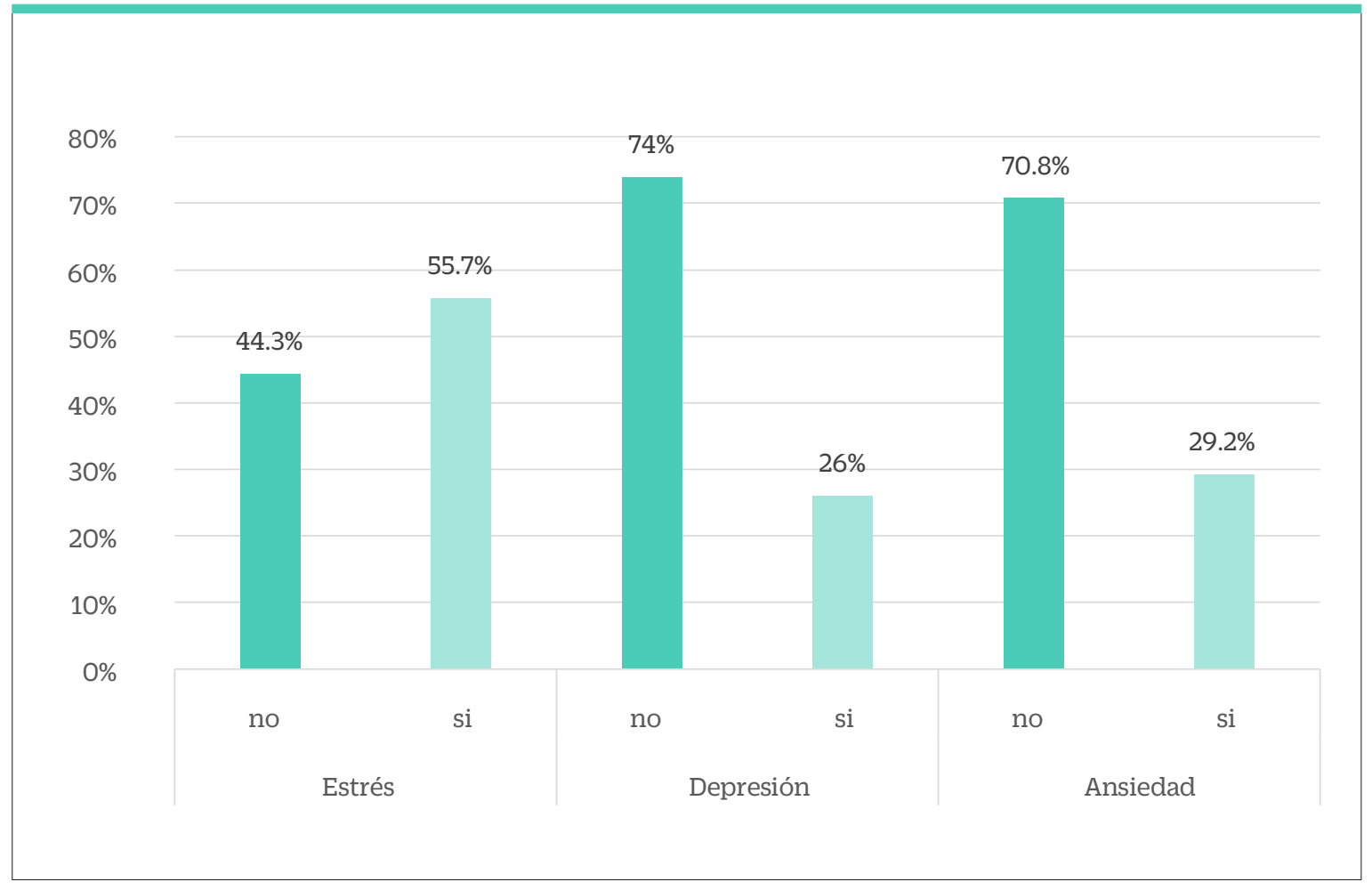

Figura 1. Presencia de Estrés, Depresión y Ansiedad en Funcionarios de una Universidad Chilena

(Chi-cuadrado=12,91; $\mathrm{p}=, 012$ ), en donde los grupos edades inferiores a 39 años son los más afectados, tendiendo a disminuir en los grupos mayores a este rango. También se observan diferencias significativas en los niveles de estrés según el tipo de contrato (Chi-cuadrado=6,405; $p=, 04$ ), observándose mayores niveles de estrés en funcionarios a contrata (contrato a plazo fijo de carácter anual), comparados con funcionarios de planta y honorarios. No se aprecian diferencias significativas en la presencia de estrés según los años de experiencia laboral en la institución (Chi-cuadrado=3,168; $\mathrm{p}=$,53) (ver detalle en Tabla 1).

No se observan diferencias en presencia de depresión según sexo (Chi-cuadrado=1,15; $p=, 284$ ), tipo de funcionario (Chi-cuadrado=,257; $p=, 612$ ), edad (Chi-cuadrado=3,216; $p=, 522$ ), años de experiencia en la institución (Chi-cuadrado=8,916; $\mathrm{p}=, 63$ ) o tipo de contrato (Chi-cuadrado=4,492; $\mathrm{p}=, 106$ ) (ver detalle en Tabla 2).

En el caso de la presencia de ansiedad, no se observan diferencias significativas según sexo (Chi-cuadrado=1,858; $p=, 173$ ), tipo de funcionario (Chi-cuadrado=1,488; $p=, 223$ ), edad (Chi-cuadra- do=5,118; $p=, 275)$, ni años de experiencia en la institución (Chi-cuadrado=5,293; $p=, 259$ ). Sin embargo, si se observa diferencias significativas según tipo de contrato (Chi-cuadrado=6,725; $p=, 035$ ), evidenciándose una mayor presencia de ansiedad en funcionarios a contrata, por sobre los funcionarios de planta y de honorarios. (ver detalle en Tabla 3)

Junto a lo anterior, se observó la relación entre las variables psicopatológicas evaluadas con agotamiento laboral y carga de trabajo percibida. Se destaca que el estrés se asociaría significativamente a mayores niveles de agotamiento laboral $(\mathrm{r}=, 775 ; \mathrm{p}<, 01)$ y carga de trabajo percibida $(\mathrm{r}=, 628$; $\mathrm{p}<, 01)$. También se observa relación entre depresión y agotamiento laboral $(r=, 62 ; \mathrm{p}<, 01)$ y carga de trabajo percibida $(r=, 474 ; p<, 01)$. De la misma manera, ansiedad estaría relacionada con mayores niveles de agotamiento laboral $(r=, 631 ; p<, 01)$ y carga de trabajo percibida $(r=, 484 ; p<, 01)$ (Ver Tabla 4).

Considerando los resultados correlacionales obtenidos y la alta presencia de estrés, se verificó un modelo predictivo de esta variable. En este 
Jorquera , R. \& Herrera, F.

Tabla 1

Presencia de Estrés según Ssexo, Tipo de Funcionario, Edad, Años de Experiencia en la Institución y Tipo de Contrato

\begin{tabular}{|c|c|c|c|c|c|}
\hline & & Prese & strés & Chi-cuadrado & $m$ \\
\hline & & No & SI & de Pearson & p \\
\hline & Hombre & $51,5 \%$ & $48,5 \%$ & 4,206 & 0,04 \\
\hline exu & Mujer & $36,8 \%$ & $63,2 \%$ & & \\
\hline$\Gamma$ & Académico(a) & $36,4 \%$ & $63,6 \%$ & 8,292 & 0,004 \\
\hline Funtivilatio(a) & No Académico(a) & $57,7 \%$ & $42,3 \%$ & & \\
\hline & 20-29 años & $38,5 \%$ & $61,5 \%$ & 12,91 & 0,012 \\
\hline & 30-39 años & $31,2 \%$ & $68,8 \%$ & & \\
\hline Edad & 40-49 años & $47,1 \%$ & $52,9 \%$ & & \\
\hline & 50-59 años & $64,7 \%$ & $35,3 \%$ & & \\
\hline & 60 años o más & $58,8 \%$ & $41,2 \%$ & & \\
\hline & 1 año o menos & $41,2 \%$ & $58,8 \%$ & 3,168 & 0,53 \\
\hline Años de & 11-20 años & $50,0 \%$ & $50,0 \%$ & & \\
\hline $\begin{array}{l}\text { experiencia } \\
\text { laboral en la }\end{array}$ & 2 a 5 años & $39,5 \%$ & $60,5 \%$ & & \\
\hline institución & 21 años o más & $60,0 \%$ & $40,0 \%$ & & \\
\hline & 6 a 10 años & $43,8 \%$ & $56,3 \%$ & & \\
\hline & Contrata & $37,3 \%$ & $62,7 \%$ & 6,405 & 0,041 \\
\hline Tipo de Contrato & Honorarios & $63,6 \%$ & $36,4 \%$ & & \\
\hline & Planta & $54,0 \%$ & $46,0 \%$ & & \\
\hline
\end{tabular}

Tabla 2

Presencia de Depresión según Sexo, Tipo de Funcionario, Edad, Años de Experiencia en la Institución y Tipo de Contrato

\begin{tabular}{|c|c|c|c|c|c|}
\hline & & Presen & presión & Chi-cuadrado & $m_{2}$ \\
\hline & & No & SI & de Pearson & $\mathbf{p}$ \\
\hline$S_{0}$ & Hombre & $77,30 \%$ & $22,70 \%$ & 1,15 & 0,284 \\
\hline JeAU & Mujer & $70,30 \%$ & $29,50 \%$ & & \\
\hline Гиmpionoripla & Académico(a) & $72,70 \%$ & $27,30 \%$ & 0,257 & 0,612 \\
\hline Funtivilartu(a) & No Académico(a) & $76,10 \%$ & $23,90 \%$ & & \\
\hline & 20-29 años & $61,50 \%$ & $38,50 \%$ & 3,216 & 0,522 \\
\hline & 30-39 años & $71,40 \%$ & $28,60 \%$ & & \\
\hline Edad & 40-49 años & $74,50 \%$ & $25,50 \%$ & & \\
\hline & 50-59 años & $76,50 \%$ & $23,50 \%$ & & \\
\hline & 60 años o más & $88,20 \%$ & $11,80 \%$ & & \\
\hline & 1 año o menos & $70,60 \%$ & $29,40 \%$ & 8,916 & 0,63 \\
\hline Años de & 11-20 años & $96,20 \%$ & $3,80 \%$ & & \\
\hline experiencia & 2 a 5 años & $67,90 \%$ & $32,10 \%$ & & \\
\hline institución & 21 años o más & $80,00 \%$ & $20,00 \%$ & & \\
\hline & 6 a 10 años & $70,80 \%$ & $29,20 \%$ & & \\
\hline & Contrata & $68,60 \%$ & $31,40 \%$ & 4,492 & 0,106 \\
\hline Tipo de Contrato & Honorarios & $81,80 \%$ & $18,20 \%$ & & \\
\hline & Planta & $82,50 \%$ & $17,50 \%$ & & \\
\hline
\end{tabular}


Tabla 3

Presencia de Ansiedad según Sexo, Tipo de Funcionario, Edad, Años de Experiencia en la Institución y Tipo de Contrato

\begin{tabular}{|c|c|c|c|c|c|}
\hline & & \multicolumn{2}{|c|}{ Presencia de Ansiedad } & \multirow{2}{*}{$\begin{array}{l}\text { Chi-cuadrado } \\
\text { de Pearson }\end{array}$} & \multirow{2}{*}{$\mathbf{p}$} \\
\hline & & NO & SI & & \\
\hline \multirow{2}{*}{ Sexo } & Hombre & $75,30 \%$ & $24,70 \%$ & 1,858 & 0,173 \\
\hline & Mujer & $66,30 \%$ & $33,70 \%$ & & \\
\hline \multirow{2}{*}{ Funcionario(a) } & Académico(a) & $67,80 \%$ & $32,20 \%$ & 1,488 & 0,223 \\
\hline & No Académico(a) & $76,10 \%$ & $23,90 \%$ & & \\
\hline \multirow{5}{*}{ Edad } & 20-29 años & $61,50 \%$ & $38,50 \%$ & 5,118 & 0,275 \\
\hline & 30-39 años & $64,90 \%$ & $35,10 \%$ & & \\
\hline & 40-49 años & $70,60 \%$ & $29,40 \%$ & & \\
\hline & 50-59 años & $82,40 \%$ & $17,60 \%$ & & \\
\hline & 60 años o más & $82.4 \%$ & $17,60 \%$ & & \\
\hline \multirow{5}{*}{$\begin{array}{l}\text { Años de } \\
\text { experiencia } \\
\text { laboral en la } \\
\text { institución }\end{array}$} & 1 año o menos & $76,50 \%$ & $23,50 \%$ & 5,293 & 0,259 \\
\hline & 11-20 años & $84,60 \%$ & $15,40 \%$ & & \\
\hline & 2 a 5 años & $64,20 \%$ & $35,80 \%$ & & \\
\hline & 21 años o más & $80,00 \%$ & $20,00 \%$ & & \\
\hline & 6 a 10 años & $68,80 \%$ & $31,30 \%$ & & \\
\hline \multirow{3}{*}{ Tipo de Contrato } & Contrata & $64,40 \%$ & $35,60 \%$ & 6,725 & 0,035 \\
\hline & Honorarios & $90,90 \%$ & $9,10 \%$ & & \\
\hline & Planta & $79,40 \%$ & $20,20 \%$ & & \\
\hline
\end{tabular}

Tabla 4

Correlaciones Estrés, Depresión, Ansiedad, Agotamiento Laboral y Carga de Trabajo Percibida

\begin{tabular}{|l|c|c|c|c|}
\hline & Depresión & Ansiedad & $\begin{array}{c}\text { Agotamiento } \\
\text { Laboral }\end{array}$ & $\begin{array}{c}\text { Carga de Trabajo } \\
\text { Percibida }\end{array}$ \\
\hline Estrés &, $795^{* *}$ &, $839^{* *}$ &, $775^{* *}$ &, $628^{* *}$ \\
\hline Depresión & &, $857^{* *}$ &, $620^{* *}$ &, $474^{* *}$ \\
\hline Ansiedad & & &, $631^{* *}$ &, $484^{* *}$ \\
\hline Agotamiento Laboral & & & &, $818^{* *}$ \\
\hline
\end{tabular}

**. La correlación es significativa en el nivel 0,01 (2 colas).

modelo, se observa que la carga de trabajo percibida logra predecir el agotamiento laboral $(\beta=, 82$; $\mathrm{p}<, 01)$, explicando un $67 \%$ de la varianza de esta variable. Además, el agotamiento laboral predice el estrés $(\beta=, 78 ; \mathrm{p}<, 01)$, explicando un $60 \%$ de su varianza. Este modelo mostró indicadores de ajustes adecuados $(\chi 2=, 065, \mathrm{gl}=1, \mathrm{p}<, 798)$.

Se generó un modelo similar en el caso de la depresión. En este caso el agotamiento laboral también logra predecir un incremento en los niveles de esta sintomatología $(\beta=, 62 ; \mathrm{p}<, 01)$, sin embargo, la varianza explicada es de un $38 \%$, menor que en el caso del estrés. Este modelo mostró buenos indicadores de ajuste $(\chi 2=1,076, g l=1, p<, 3)$.

De igual forma, se probó un modelo predictivo para la sintomatología ansiosa. En este modelo, se observa que el agotamiento laboral predice la ansiedad $(\beta=, 63 ; \mathrm{p}<, 01)$, explicando un $40 \%$ de su varianza. Este modelo también mostró indicadores de ajustes adecuados $(\chi 2=1,018, g l=1, p<, 313)$. 


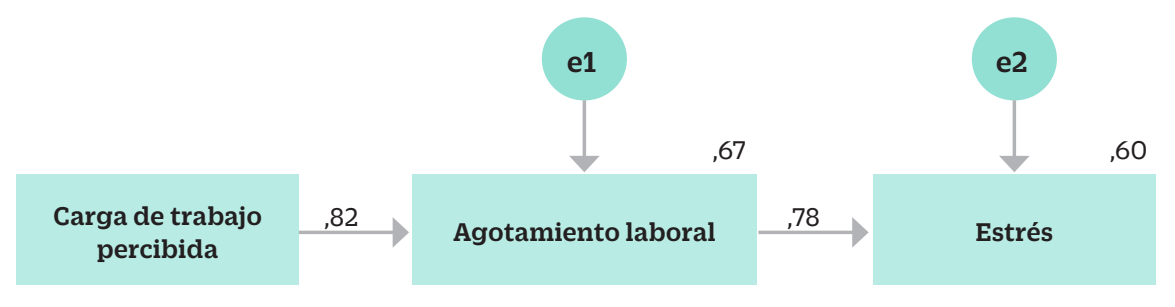

Figura 2. Modelo Predictivo de Estrés, Agotamiento Laboral y Carga de Trabajo Percibida

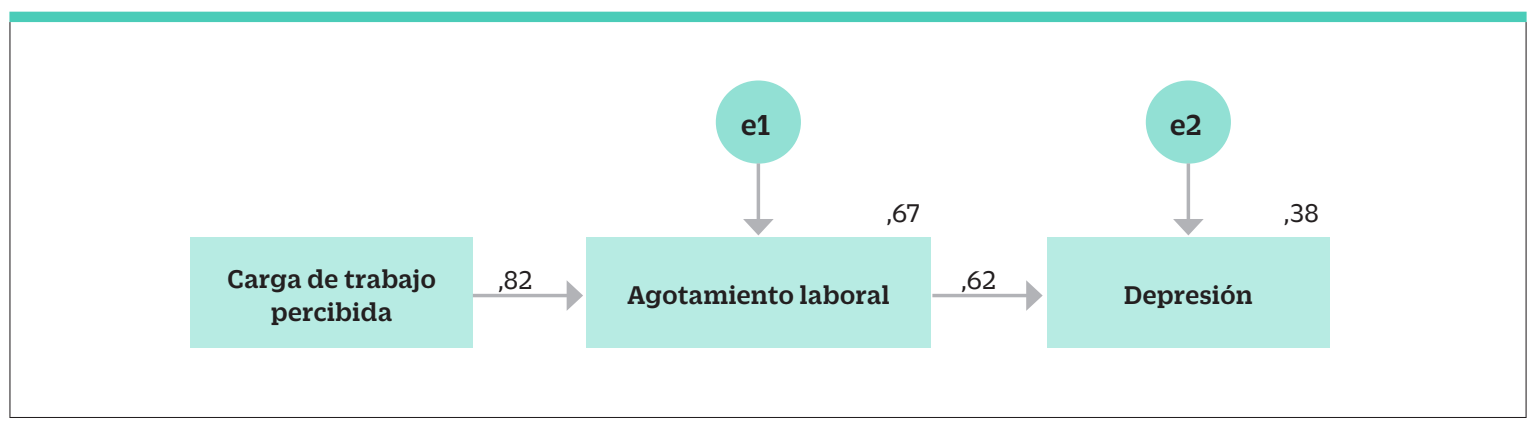

Figura 3. Modelo Predictivo de Depresión, Agotamiento Laboral y Carga de Trabajo Percibida

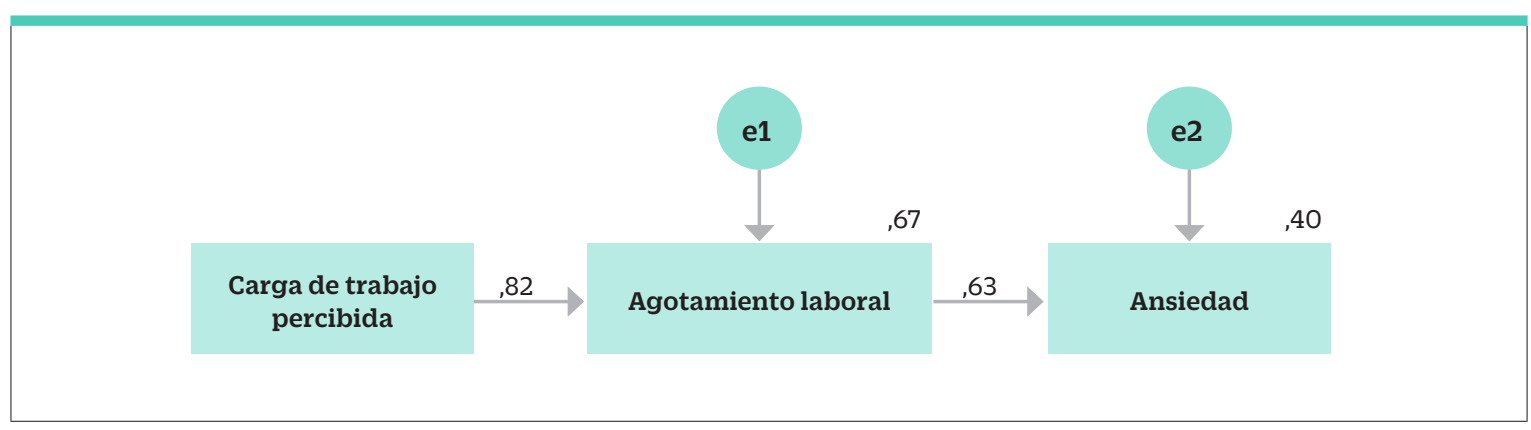

Figura 4. Modelo Predictivo de Ansiedad, Agotamiento Laboral y Carga de Trabajo Percibida

\section{Discusión}

Los resultados evidencian que una proporción mayoritaria de los funcionarios universitarios presentarían altos niveles de estrés, siendo la afección psicológica más importante dentro de las tres evaluadas. Esta problemática afectaría de forma más importante a académicos, lo cual se podría explicar por el acomodo de buena parte de su funcionamiento docente a un formato de teleducación, en un contexto en el cual el $80 \%$ de ellos no tenía experiencia previa en esta modalidad laboral. A ello, es necesario sumar que las condiciones actuales significan ejecutar este tipo de labores conciliándola con roles familiares y en condiciones estructurales que no siempre son óptimas. Esta realidad es compartida con buena parte de los trabajadores que han debido pasar del trabajo presencial al de tipo remoto a través de las tecnologías de la información, a quienes también les ha significado un aumento en horas de trabajo, la realización de las labores en espacios que no siempre están acondicionados para ello y, en muchos 
casos, sin las herramientas informáticas (computador e internet) que permiten una adecuada conectividad. Junto a ello, los trabajadores han debido compatibilizar su quehacer con los asuntos del hogar y la convivencia familiar (Circular HR, 2020). Esto ha hecho que la labor académica no haya estado exenta de dificultades.

Los resultados evidencian un mayor nivel de estrés en mujeres. Esto es consistente con investigaciones que han mostrado un mayor deterioro de la salud mental en ellas (Gao et al., 2020; Ozamiz-Etxebarria et al., 2020; Vela et al., 2020; Wang et al., 2020), y con estudios en trabajadoras chilenas que han evidenciado menores niveles de engagement y mayores niveles de agotamiento (Circular HR, 2020), lo cual se explicaría por la acentuación, en este contexto, de la multiplicidad de roles que ellas cumplen. En este sentido, en contextos de aislamiento social, se hace más fuerte el peso de tener que responder al trabajo y conciliarlo con las labores en el hogar y de crianza. Además, hace patente nuevamente que, si bien en las últimas décadas las mujeres han tenido un mayor acceso al espacio público y al mercado laboral, en el espacio domestico siguen asumiendo buena parte de las responsabilidades (PNUD, 2010). Esto llama a generar acciones orientadas a disminuir el estrés entre los funcionarios universitarios incorporando una perspectiva de género.

Otro resultado relevante es que los funcionarios jóvenes (de menos de cuarenta años) manifiestan mayores niveles de estrés que los funcionarios de mayor edad, lo cual es consistente con la literatura al respecto, que ha mostrado mayor compromiso de la salud mental en jóvenes (Ozamiz-Etxebarria et al., 2020; Wang et al., 2020), pero también con estudios en contextos universitarios que han mostrado que profesores de menor edad y de menor antigüedad en la institución manifestarían mayores niveles de cansancio emocional y despersonalización, comparado con profesores de mayor edad (Sánchez \& Clavería, 2005). Posiblemente, contextos de vida menos estructurados y en proceso de desarrollo llevan a acentuar los niveles de incertidumbre que provocan realidades inciertas, como las que se viven producto del contexto presente.

Los resultados también muestran que los funcionarios en una modalidad a contrata (contrato laboral a plazo fijo de carácter anual) manifiestan mayores niveles de estrés y ansiedad, comparado con funcionarios de planta y honorarios. Esta realidad ya había sido expuesta por Cladellas-Pros, Castelló-Tarrida y Parrado-Romero (2018) quienes mostraron que profesores universitarios de tiempo completo con contratos laborales temporales muestran una peor salud física y mental, mayores síntomas de estrés y una menor satisfacción laboral, en comparación con profesores a tiempo completo y contrato estable o profesores de tiempo parcial. Pero, además, es posible plantear como línea interpretativa que la incertidumbre del contexto afectaría especialmente a este tipo de trabajadores, dado que se hace presente el temor al desempleo y a la no continuidad del contrato, considerando las posibles necesidades económicas que pudiese llegar a tener la institución, a propósito de la situación económica del país. Al respecto, se recomien$\mathrm{da}$, a quienes ocupan funciones de liderazgo, mantener relaciones cercanas con sus supervisados y favorecer la disminución de la incertidumbre laboral provocada por la situación actual.

Los resultados permiten apreciar la relación entre variables asociadas a salud psicológica y variables laborales en los funcionarios. Especialmente, se logra observar que mayores niveles de agotamiento producto del trabajo permiten predecir mayores niveles de afección psicológica, y muy especialmente, mayores niveles de estrés. Lo anterior, pone de relieve considerar lo que múltiples autores han mostrado respecto de la importancia de los factores de riesgo psicosociales para la salud y calidad de vida de los trabajadores (Solanova \& Llorens, 2009; Gil-Monte, 2012), pero contextualizados en una realidad histórica como la que se encuentra viviendo el mundo.

Considerando lo anterior, en el ámbito de la gestión de instituciones de educación superior, se recomienda generar acciones en tres direcciones:

Primero, es recomendable atender a la formalización de procesos que den estructura al trabajo de académicos y no académicos en un contexto especial como el de la pandemia. Es prudente la flexibilización de ellos, sin embargo, estos deben ser claros y conocidos por los implicados. En este sentido, tener objetivos y procedimientos predecibles, ayuda a disminuir los niveles de incertidumbre que favorecen el surgimiento de estrés y otras afecciones en el contexto laboral. Esto se 
condice con evidencias que muestran que incrementos en la claridad de rol percibida producen una disminución de la tensión laboral experimentada, y, de la misma forma, niveles elevados de esta variable conllevan también niveles elevados de satisfacción laboral (Bravo, Zurriaga, Peiró \& Gonzalez, 1993).

En segundo lugar, es muy importante la figura del liderazgo. Es necesario reconocer que el liderazgo cumple un importante papel de apoyo social en contextos actuales, favoreciendo, por un lado, claridad respecto de los objetivos, metas y procedimientos de trabajo, los cuales deberían, como ya se señaló, ajustarse a las actuales condiciones de trabajo. Por otro lado, el apoyo del líder debe enfocarse también a temáticas afectivas, por tanto, se recomienda que ellos generen una relación cercana con sus equipos, idealmente generando una consideración individual con cada trabajador, y siendo capaz de contener emocionalmente a los funcionarios más afectados por la situación actual. Estos aspectos son coherentes con la aplicación de un estilo de liderazgo transformacional (Bass,1997), dado que el líder debería tener en cuenta individualmente los requerimientos técnicos y cognitivos del trabajador, pero a su vez, reconociendo y atendiendo sus necesidades en el plano emocional y afectivo. Bajo las condiciones provocadas por la pandemia, considerando los esfuerzos que ha demandado impartir la docencia universitaria en formato de educación remota, se hace muy importante realizar reconocimiento a los docentes y otorgarles incentivos que honren sus esfuerzos (Vicentini, 2020).

En tercer lugar, es recomendable favorecer el conocimiento y el desarrollo de estrategias de afrontamiento contra el estrés en los funcionarios. Esto debería tener en cuenta especialmente las consecuencias del estrés provocado por esta forma de trabajo. Por ello, es imprescindible implementar herramientas para desarrollar el trabajo remoto en formato telemático, pero, además, considerando el efecto que está teniendo el conflicto trabajo-familia sobre este formato de trabajo en el contexto actual. Es relevante considerar que el teletrabajo genera una superposición entre el trabajo remunerado y la vida personal (Organización Internacional del Trabajo, 2019). El efecto del teletrabajo sobre la vida personal se incrementaría cuando el trabajador no tiene un espacio exclusivo para realizar sus labores y cuando hay más personas en la casa mientras se trabaja (Salazar, 2016), lo cual es la realidad del trabajo académico en estos días de confinamiento.

\section{Referencias}

Antúnez, Z., \& Vinet, E. V. (2012). Escalas de Depresión, Ansiedad y Estrés (DASS - 21): Validación de la Versión abreviada en Estudiantes Universitarios Chilenos. Terapia psicológica, 30(3), 49-55. https:// doi.org/10.4067/S0718-48082012000300005

Bass, B. M. (1997). Does the transactional-transformational leadership paradigm transcend organizational and national boundaries? American Psychologist, 52(2), 130-139. https://doi.org/10.1037/0003-066X.52.2.130

Vicentini, I. (2020). La Educación Superior en Contextos de Covid-19: Aportes de la Segunda Reunión del Dialogo Virtual con Rectores de Universidades Líderes de América Latina. Washington DC: Banco Interamericano de Desarrollo

Bravo, M., Zurriaga, R., Peiró, J. \& González, P. (1993). Un Modelo Causal sobre los Antecedentes y Consecuentes del Estrés de Rol en Profesionales de la Salud. Revista de Psicología de la Salud, 5(1), 19 36

Circular HR. (2020). Encuesta "Engagement y Teletrabajo en contexto COVID-19". https://www.circularhr.cl/ noticias/encuesta-engagement-y-teletrabajo-encontexto-covid-19-un-40-de-las-personas-senalanque-estan-cumpliendo-sus-tareas-laborales-depeor-forma-que-antes/

Cladellas-Pros, R., Castelló-Tarrida, A. \& Parrado-Romero, E. (2018). Satisfacción, salud y estrés laboral del profesorado universitario según su situación contractual. Revista de Salud Pública, 20 (1), 53-59. https://dx.doi.org/10.15446/rsap.v20n1.53569

Cuadra Martínez, D., Jorquera Gutiérrez, R., \& Pérez Cea, M. (2015). Las teorías subjetivas del profesor acerca de su salud laboral: Implicancias en la promoción de la salud preventiva en el trabajo docente. Ciencia \& Trabajo, 17(52), 1-6. https://doi.org/10.4067/S0718 24492015000100002

Gao, J., Zheng, P., Jia, Y., Chen, H., Mao, Y., Chen, S., Wang, Y., Fu, H., \& Dai, J. (2020). Mental health problems and 
social media exposure during COVID-19 outbreak. PLOS ONE, 15(4), e0231924. https://doi.org/10.1371/ journal.pone.0231924

García, M. I. V. de V., \& Gambarte, M. I. G. (2019). La resiliencia como factor protector del estrés crónico en docentes. European Journal of Investigation in Health, Psychology and Education, 9(3), 159-175. https://doi.org/10.30552/ejihpe.v9i3.332

Gil-Monte, P. (2012). Riesgos psicosociales en el trabajo y salud ocupacional. Revista Peruana de Medicina Experimental y Salud Publica, 29(2), 237-241. http://www.scielo.org.pe/scielo.php?script=sci arttext\&pid=S1726-46342012000200012\&lng=es\&t $\operatorname{lng}=\mathrm{es}$

Guerrero-Barona, E., Gómez Del Amo, R., Moreno-Manso, J., \& Guerrero-Molina, M. (2018). Factores de riesgo psicosocial, estrés percibido y salud mental en el profesorado. Clínica Contemporánea, 9(1), E2, 1-12. https://doi.org/10.5093/cc2018a2

Hernández, R., Fernández, C., \& Baptista, P. (2014). Metodología de la Investigación. México: McGraw Hill.

Hernández, R. \& Mendoza, C. (2018). Metodología de la investigación: las rutas cuantitativa, cualitativa y mixta. México: McGraw Hill.

Ilaja, B. \& Reyes, C. (2016). Burnout y estrategias de inteligencia emocional en profesores universitarios: implicaciones en la salud laboral educativa. Psicología desde el Caribe, 33(1), 31-46

Jorquera-Gutiérrez, R., Orellana Herrera, C., Tapia Núñez, C., \& Vergara Magnata, E. (2014). Síndrome de Burnout en una muestra de profesores/as de enseñanza básica de la ciudad de Copiapó. Summa Psicológica UST, 11(2), 115-134.

Mann, S., \& Holdsworth, L. (2003). The psychological impact of teleworking: Stress, emotions and health. New Technology, Work and Employment, 18(3), 196-211. https://doi.org/10.1111/1468-005X.00121

Moore, J. E. (2000). One Road to Turnover: An Examination of Work Exhaustion in Technology Professionals. MIS Quarterly, 24(1), 141-168. JSTOR. https://doi. org/10.2307/3250982

Organización Internacional del Trabajo (2019). Trabajar en cualquier momento $y$ en cualquier lugar: Consecuencias en el ámbito laboral [Publicación]. http://www.ilo.org/santiago/WCMS_723962/lang-es/index.htm

Ozamiz-Etxebarria, N., Dosil-Santamaria, M., PicazaGorrochategui, M., \& Idoiaga-Mondragon, N.
(2020). Stress, anxiety, and depression levels in the initial stage of the COVID-19 outbreak in a population sample in the northern Spain. Cadernos De Saude Publica, 36(4), e00054020. https://doi. org/10.1590/0102-311X00054020

PNUD. (2010). Desarrollo Humano en Chile. Género: Los desafíos de la igualdad. Programa de las Naciones Unidas para el Desarrollo. https://www.cl.undp. org/content/chile/es/home/library/human development/publication_2.html

Rodríguez-Martínez, M., Tovalin-Ahumada, J., Gil-Monte, P., Salvador-Cruz, J. \& Acle-Tomasini G. (2018) Trabajo Emocional y Estresores Laborales como Predictores de Ansiedad y Depresión en Profesores Universitarios Mexicanos. Información Psicológica, 115, 93-107. http://dx.medra.org/10.14635/ IPSIC.2018.115.11

Román, F., Santibáñez, P., \& Vinet, E. V. (2016). Uso de las Escalas de Depresión Ansiedad Estrés (DASS21) como Instrumento de Tamizaje en Jóvenes con Problemas Clínicos1. Acta de Investigación Psicológica - Psychological Research Records, 6(1), 2325-2336. https://doi.org/10.1016/S20074719(16)30053-9

Rubio-González, J., Andrade Daigre, P., Fravega Araneda, G., Macalusso Salgado, S., y Soto Sandoval, A. (2019). Factores psico-socio-ambientales asociados al estrés laboral en profesores chilenos del ámbito rural y urbano. Propósitos y Representaciones, 7(3), 300-311. https://doi.org/10.20511/pyr2019.v7n3.358

Salanova, M. \& Llorens, S. (2009). Presente y futuro de la intervención profesional en factores psicosociales. Gestión Práctica de Riesgos Laborales, 59, 20-23. http://www.want.uji.es/wp-content/ uploads/2017/06/2009_Salanova-Llorens.pdf

Salazar, M. (2016). Telework: Conditions that have a positive and negative impact on the work-family conflict. Academia Revista Latinoamericana de Administración, 29(4), 435-449. https://doi. org/10.1108/ARLA-10-2015-0289

Sánchez, M. \& Clavería, M. (2005). Profesorado Universitario: Estrés Laboral. Factor de Riesgo de Salud. Enfermería Global, 4(1), 1-16. https://doi. org/10.6018/eglobal.4.1.512

Vela, M. F., Rossi, D., Sánchez, J. L., Quiero Carrión, A., Mesa, Z., Lucero, Y., Ragone, F. A., Jofré Neila, M., Maschi, C., y Belamate, C. (2020). La Relajación Percibida y su Rol Como Factor Protector Frente a la Ansiedad Fóbica Durante el Transcurso del Aislamiento 
Social, Preventivo y Obligatorio por Covid-19 en el Gran Mendoza [Preprint]. https://doi.org/10.1590/ SciELOPreprints.82

Wang, C., Pan, R., Wan, X., Tan, Y., Xu, L., Ho, C. S., \& Ho, R. C. (2020). Immediate Psychological Responses and Associated Factors during the Initial Stage of the 2019 Coronavirus Disease (COVID-19) Epidemic among the General Population in China. International Journal of Environmental Research and Public Health, 17(5). https://doi.org/10.3390/ ijerph17051729

RIDU / Revista Digital de Investigación en Docencia Universitaria / e-ISSN: 2223-2516

(C) Los autores. Este artículo es publicado por la Revista Digital de Investigación en Docencia Universitaria del Área de Institutional Research and Effectiveness de la Dirección de Aseguramiento de la Calidad, Universidad Peruana de Ciencias Aplicadas. Este es un artículo de acceso abierto, distribuido bajo los términos de la LicenciaCreativeCommons Atribución-CompartirIgual 4.0 Internacional.( http://creativecommons.org/licenses/by-sa/4.0/), que permite el uso no comercial, distribución y reproducción en cualquier medio, siempre que la obra original sea debidamente citada. 